\title{
Strengthening organizational performance through accreditation research-a framework for twelve interrelated studies: the ACCREDIT project study protocol
}

\author{
Jeffrey Braithwaite ${ }^{1 *}$, Johanna Westbrook ${ }^{1}$, Brian Johnston ${ }^{2}$, Stephen Clark ${ }^{3}$, Mark Brandon ${ }^{4}$, Margaret Banks ${ }^{5}$, \\ Clifford Hughes ${ }^{6}$, David Greenfield ${ }^{1}$, Marjorie Pawsey², Angus Corbett ${ }^{7}$, Andrew Georgiou', Joanne Callen', \\ John Vvretveit $^{8}$, Catherine Pope ${ }^{9}$, Rosa Suñol ${ }^{10}$, Charles Shaw ${ }^{11}$, Deborah Debono ${ }^{1}$, Mary Westbrook', \\ Reece Hinchcliff ${ }^{1}$ and Max Moldovan ${ }^{1}$
}

\begin{abstract}
Background: Service accreditation is a structured process of recognising and promoting performance and adherence to standards. Typically, accreditation agencies either receive standards from an authorized body or develop new and upgrade existing standards through research and expert views. They then apply standards, criteria and performance indicators, testing their effects, and monitoring compliance with them. The accreditation process has been widely adopted. The international investments in accreditation are considerable. However, reliable evidence of its efficiency or effectiveness in achieving organizational improvements is sparse and the value of accreditation in cost-benefit terms has yet to be demonstrated. Although some evidence suggests that accreditation promotes the improvement and standardization of care, there have been calls to strengthen its research base.

In response, the ACCREDIT (Accreditation Collaborative for the Conduct of Research, Evaluation and Designated Investigations through Teamwork) project has been established to evaluate the effectiveness of Australian accreditation in achieving its goals. ACCREDIT is a partnership of key researchers, policymakers and agencies.

Findings: We present the framework for our studies in accreditation. Four specific aims of the ACCREDIT project, which will direct our findings, are to: (i) evaluate current accreditation processes; (ii) analyse the costs and benefits of accreditation; (iii) improve future accreditation via evidence; and (iv) develop and apply new standards of consumer involvement in accreditation. These will be addressed through 12 interrelated studies designed to examine specific issues identified as a high priority. Novel techniques, a mix of qualitative and quantitative methods, and randomized designs relevant for health-care research have been developed. These methods allow us to circumvent the fragmented and incommensurate findings that can be generated in small-scale, project-based studies. The overall approach for our research is a multi-level, multi-study design.

Discussion: The ACCREDIT project will examine the utility, reliability, relevance and cost effectiveness of differing forms of accreditation, focused on general practice, aged care and acute care settings in Australia. Empirically, there are potential research gains to be made by understanding accreditation and extending existing knowledge; theoretically, this design will facilitate a systems view of accreditation of benefit to the partnership, international research communities, and future accreditation designers.

"Accreditation of health-care organisations is a multimillion dollar industry which shapes care in many countries. Recent reviews of research show little evidence that accreditation increases safety or improves quality. It's time we knew about
\end{abstract}

\footnotetext{
* Correspondence: j.braithwaite@unsw.edu.au

'University of New South Wales, Kensington, NSW 2052, Australia

Full list of author information is available at the end of the article
} 
the cost and value of accreditation and about its future direction." [Professor John Øvretveit, Karolinska Institute,

Sweden, 7 October 2009]

\section{Background}

Service accreditation is a system of organizational improvement centred on a certifying agency (or accrediting body) assessing performance against pre-determined standards, usually by multiple means. Internationally, accreditation is designed to improve organizations by developing new standards or upgrading existing standards through research or expert advice, and by defining criteria and performance indicators and applying these standards, criteria and indicators to organizational processes and outcomes. Although models differ in detail, [1] most accreditation systems assess and rate the performance of organizations and services by evaluating their progress and appraising their compliance with standards, usually via mechanisms such as self-assessment surveys, data review and structured visits by surveyors. Some systems use peer surveyors and others persons whose background is audit methodology. Following training, assessors or surveyors have detailed knowledge of applicable standards. Figure 1 provides a generic accreditation model which illustrates a typical accreditation process from standards development into the cycle of standards application, assessment and award of accreditation and periodic review.

The reach of accreditation is extensive and the investments in it in many sectors are considerable. Industries such as school education, [2] universities, [3,4] software manufacture, [5] the seafood sector, [6] and ambulance services [7] have embraced accreditation, standard setting, and surveying processes. Accreditation has been applied to laboratories, [8] management systems, [9] products,[10] medical curricula, [11] and staff competencies [12]. Essentially, the core concerns addressed by the processes of accreditation are whether organizations satisfy pre-designated standards, are regularly examined and continuously improved, and the extent to which customer satisfaction is met or enhanced [13].

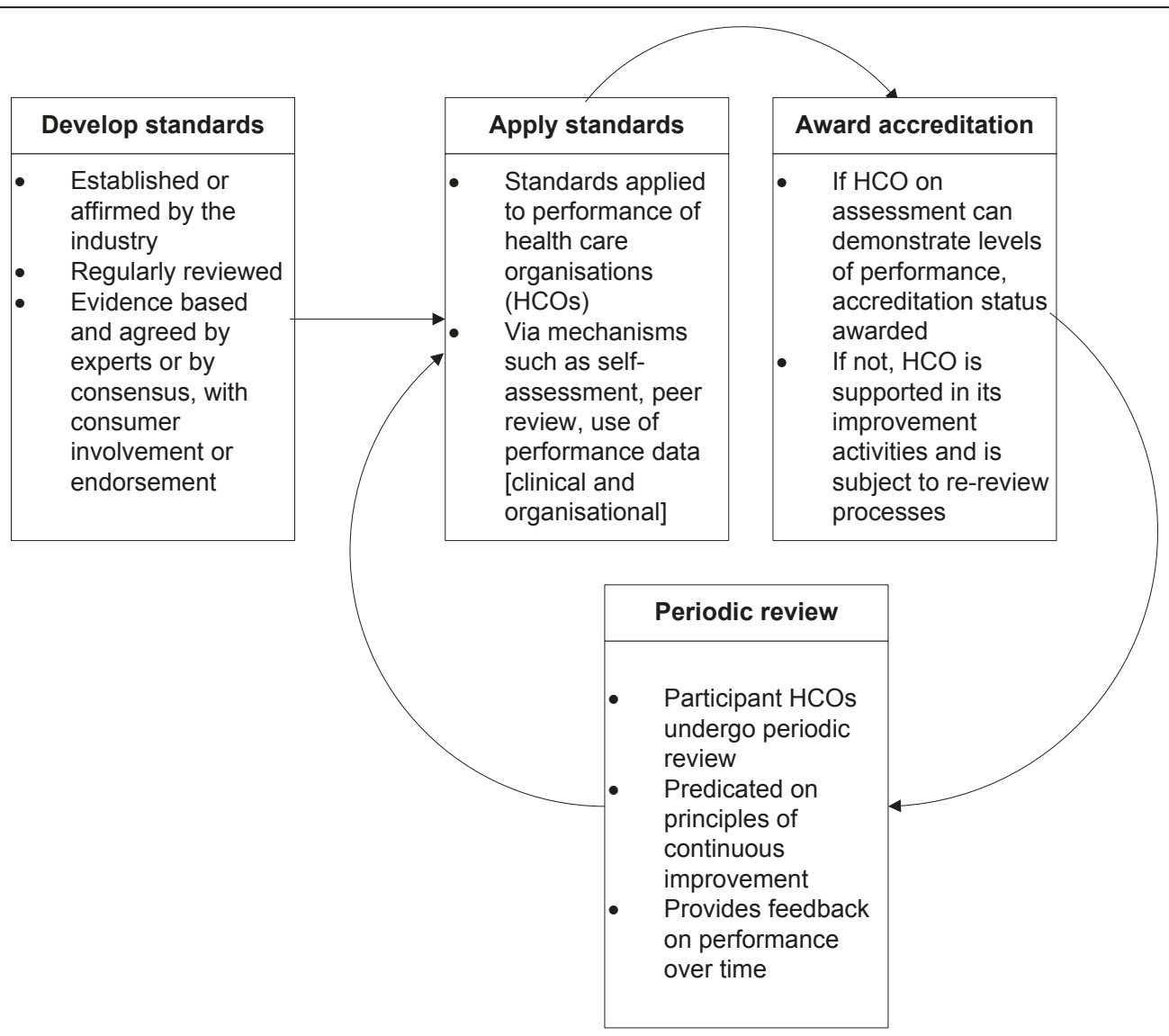

Figure 1 Generic accreditation model 
However, we lack convincing evidence of the longterm effects and organizational impact of accreditation processes.

The health sector, as an early adopter of accreditation, has promoted its use since 1951 (the Joint Commission in the United States of America), and in Australia since 1973. Stakeholders recognize its potential to improve organizational performance, quality of care, safety standards and consumer satisfaction. However, despite support for accreditation among informed groups, accreditation has had its share of criticism, including the lack of supporting evidence and concerns about the costs of uncertain benefits and whether it offers value for money [14-16]. There is a desire among stakeholders to strengthen the research base. This project is a response to the need for a program of research into accreditation that links the key industry partners and policy bodies with interested researchers, and plans to produce results which will link with other multi-method, multi-phased studies underway in Europe [17].

The ACCREDIT (Accreditation Collaborative for the Conduct of Research, Evaluation and Designated Investigations through Teamwork) project is a partnership led by researchers in the Centre for Clinical Governance Research and Centre for Health Systems and Safety Research in the Australian Institute of Health Innovation (AIHI) at University of New South Wales with the three major Australian health-sector accreditation agencies (The Australian Council on Healthcare Standards [ACHS], Australian General Practice Accreditation Limited [AGPAL], and Aged Care and Standards Accreditation Agency [ACSAA]), the leading quality improvement policy bodies (the Australian Commission on Safety and Quality in Health Care [ACSQHC] and the Clinical Excellence Commission [CEC]), key Australian investigators, and international collaborators. These partners are dedicated to studying the impact of accreditation and to executing an extended research program, to provide evidence and empirical models for ways in which accreditation can be improved.

\section{Research significance and importance of the problem}

Some evidence suggests that accreditation programs can promote change [18] and the standardization of services and organizational processes, including how decisions about care are made [19]. However, the research literature is either inconsistent or does not support the contentions that accreditation directly improves organizational performance, quality of care, and patient satisfaction [14,20-22]. In one of the first studies to attempt to link accreditation with organizational outcomes, we found that accreditation was significantly positively correlated with organizational culture $(P=$ $0.005)$ and leadership $(P=0.005)$, but there was weaker statistical evidence on the relation to clinical indicator performance $(P=0.080)$ [23]. No statistically significant association was observed between accreditation and organizational climate $(P=0.110)$ or consumer involvement $(P=0.377)$ [23].

Thirty-four of 89 selected hospitals in the European Methods of Assessing Response to Quality Improvement Strategies (MARQuIS) project [24] were accredited (without International Organization for Standardization (ISO) certification), 10 were ISO9000-certificated without accreditation and 27 had neither accreditation nor certification. On 229 criteria of quality and safety, percentage scores were $66.9,60.0$ and 51.2 respectively. These statistically significant differences suggest that accreditation is a key quality strategy. However, there were confounding factors and a small sample, and the study did not substantially differentiate between accreditation and certification only [25].

To date, work on the costs and benefits of accreditation has been rudimentary [26-28]. Unless the economic benefits are modelled, we cannot make sound policy decisions about the future enhancement of accreditation, develop a new framework for its conduct, or understand its value.

In preparatory work to develop the framework reported here, we examined the literature concerning two initiatives that have recently received policy support: unannounced (short-notice) surveys conducted by surveyors [29] and tracer methodology (i.e., patient journeys) used to assess care [30]. We found no evidence for the benefits of short-notice surveys, whereas the limited studies of patient journeys suggested that they can be useful in evaluating care. Work commissioned by ACSQHC, and undertaken by ACHS, ACSAA and AGPAL in conjunction with ACCREDIT researchers assessing these short-notice surveys and patient journeys trials tentatively indicated that these can be useful tools which complement but do not substitute for existing methods.

A systematic review of the literature conducted by the research team "... reveals a complex picture ... inconsistent findings ... [and] ... insufficient studies by which to draw conclusions."[[14] p.181] An overarching research framework with twelve interrelated studies (Figure 2) aims to address some of these gaps.

\section{Methods and design \\ General aims}

We are funded to execute a multi-method, triangulated research agenda with 12 studies designed by the ACCREDIT stakeholders. The ACCREDIT partners met in August 2007 to draft the conceptual framework and research plan. They subsequently refined the plan and conducted various studies, [14,20,23,26,31-33] evaluation 


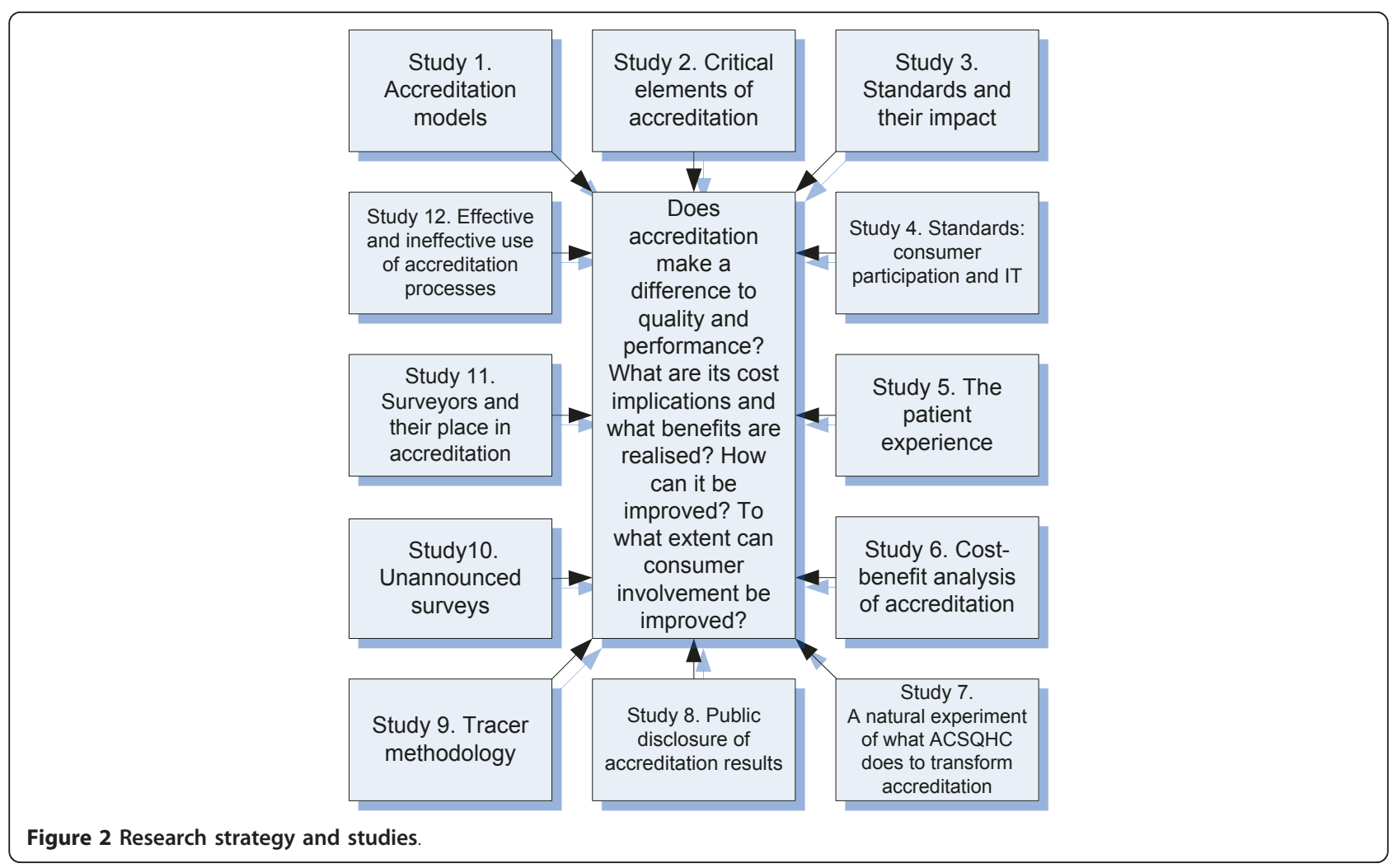

projects, $[29,30]$ literature reviews, $[21,34,35]$ and partnership activities [36] to provide the empirical platforms for this proposal. An International Advisory Group offered strategic counsel to the project, and has an ongoing advisory role.

\section{Detailed research aims}

The specific aims of the research address four main areas. These link 12 interrelated studies of issues identified as requiring research evidence as a high priority [14] (Table 1).

\section{Advancing the knowledge base}

The research aims require a multi-method, [37] multilevel approach, [38] incorporating multi-layered data, [39] to provide rigorous answers to the key questions mapped to the four research aims (Table 1) and addressed in the 12 studies (Figure 1). The 12 studies are designed to answer questions to advance the accreditation knowledge base and meet expressed industry needs for empirical information. The proposal's research questions have emerged from extensive reviews $[14,21,34]$ and consultations. Table 2 outlines the 12 interrelated studies that will address key derived questions, linking the 12 studies into four research aims.

\section{Methods, sample sizes and design features}

The samples for the quantitative studies will be based on sample size calculations that ensure sufficient power to answer the questions under investigation. Qualitative studies will involve sample sizes based on saturation methods.

Table 1 Research aims, key questions, and related studies

\begin{tabular}{|c|c|c|}
\hline Research aims & Key questions & $\begin{array}{l}\text { Studies (Fig. } \\
\text { 1) }\end{array}$ \\
\hline 1: To evaluate current accreditation processes & $\begin{array}{l}\text { Does accreditation make a difference to the quality of care and } \\
\text { performance? }\end{array}$ & $\begin{array}{l}1,2,3, \text { and } \\
12\end{array}$ \\
\hline 2: To analyse the costs and benefits of accreditation & $\begin{array}{l}\text { What are accreditation's cost implications? What benefits are } \\
\text { realized? }\end{array}$ & 6 \\
\hline 3: To improve future accreditation & How can accreditation be improved? & $7-11$ \\
\hline $\begin{array}{l}\text { 4: To develop and apply new standards of consumer involvement } \\
\text { in accreditation }\end{array}$ & How can consumer involvement be improved? & 4 and 5 \\
\hline
\end{tabular}


Table 2 Twelve interrelated studies of accreditation-overview of approaches and methods

\begin{tabular}{lll}
\hline Study & Research questions & $\begin{array}{l}\text { Research approaches, tasks, and } \\
\text { scope }\end{array}$ \\
\hline 1. Accreditation models & $\begin{array}{ll}\text { What are the relative strengths and } \\
\text { consequences of different } \\
\text { accreditation models? }\end{array}$ & $\begin{array}{l}\text { Undertake a multi-method evaluation } \\
\text { of three accreditation models: those } \\
\text { of the ACHS, AGPAL, and ACSAA }\end{array}$
\end{tabular}

Methods, sample sizes, data requirements, analysis, design features

- Interview key stakeholders in three accreditation agencies $(n=18)$

- Conduct a web-based questionnaire survey of acute health services, general practices and aged-care facilities $(\sim n=300)$

2. Critical elements of accreditation
What are the critical elements of the accreditation process that stimulate improvement? What drives behaviour change in provider organizations and clinicians?
Assess each element (e.g., selfassessment, clinical indicators, patient data, surveyor visits and accreditation reports) and describe its role in promoting improvement

- Run focus groups of stakeholders drawn from accreditation agencies ( $n$ $=6$ focus groups) and jurisdictional health departments ( $n=8$ focus groups), and 15 randomized focus groups from accredited general practices $(n=5)$, acute-care healthcare organizations $(n=5)$, and agedcare providers $(n=5)$

- Conduct a ranking exercise by surveying large samples of staff from accredited organizations across acute, general practice, and aged-care settings $(\sim n=600)$ to ascertain the relative importance of the accreditation elements

- From this sample, gather perspectives on and examples of how the respective accreditation elements drive change

3. Standards and their impact

\author{
How are standards developed and \\ used? How do standards incorporate \\ evidence, and influence the expertise \\ of clinicians, managers, and policy \\ makers? How does the application of \\ standards promote change in \\ organizational performance and \\ clinical practice?
}

Examine the development of standards and their application using widespread observational activities and surveys across different accreditation programmes, selecting and investigating a sample of standards during their development phase to determine the sources of the standards (e.g., public inquiries, adverse events, international guidelines), how they should be developed, and how they should be applied
- Undertake ethnographic observations of the development of standards, assessing their use of evidence and the engagement of stakeholder groups

- Conduct a survey of accredited organizations, investigating how standards are applied and how they promote change $(\sim n=600$ respondents). From these data, identify for detailed analysis case study sites in which standards have promoted measurable change - Conduct case studies $(n=5)$ of specified key standards (evaluation of care, documented policies, the quality improvement system, health records, infection control). Use these case studies to identify factors related to organizational change. Obtain organizational data to quantify the extent of this change

\begin{tabular}{ll}
\hline 4. Key new standard for & Can we develop and trial a standard \\
consumer participation & for consumer participation?
\end{tabular}
Use the Delphi method to create and - Systematically review instances of field test a standard for consumer participation in acute settings, general accreditation literature practice, and aged care
consumer participation cited in the
- Assess the review's evidence as the basis for the standard
- Consult with stakeholders, using the Delphi approach, to secure agreement on the standard
- Apply the standard in the field $(n=$ 30)
- Evaluate its use and efficacy with survey and qualitative methods




\section{Table 2 Twelve interrelated studies of accreditation-overview of approaches and methods (Continued)}

\author{
5. The patient experience How do patient experiences vary \\ across a range of settings with \\ differing accreditation results?
}

Compare the ethnographic mapping results for a range of patient experiences in different accreditation settings against positive and negative accreditation results
- Extend the research into patient journeys to a larger trial that includes all three accreditation domains - Review 60 randomly selected complex patient journeys in depth - Compare these against the accreditation results from each of the participating organizations in which patient journeys were taken.

6. Cost-benefit analysis of What are the benefits and costs of Design and apply a model for a health-economics cost-benefit analysis (CBA) of accreditation, including an examination of the factors that affect (e.g., drive or inhibit) costs and

- Conduct a detailed analysis of the accreditation and the different accreditation models? cost effectiveness of accreditation in the three domains, examining the extent to which the benefits outweigh the costs over time, modelled on Brent [42]

- Express the benefits and costs in nett present value terms, and adjust for the time value of money - Seek cost estimates from the partner agencies, and benefit estimates from a randomized sample ( $n=30$ ) of their member organizations

- Execute CBA modelling on this basis

7. A natural experiment examining what the ACSQHC does to transform accreditation
What changes have ensued from the initiatives of the ACSQHC?

Conduct a formative evaluation of the impact of ACSQHC's transformation of accreditation, particularly the impact of the comprehensive set of National Safety and Quality Healthcare Standards applied to high-risk services

- Evaluate this progress using

formative evaluation techniques, in a partnership arrangement with ACSQHC

- Review the gains made in establishing a national co-ordinating body to implement accreditation reforms, including standards development, piloting implementation, expanding accreditation into high-risk services, and co-ordination with regulating bodies

\begin{tabular}{ll}
\hline 8. Public disclosure of & Is it possible to develop and test an \\
accreditation results & effective model for the public
\end{tabular}

Examine extant methods of public disclosure of information in international contexts, and their relative impacts
- Identify in a literature review the different models of public disclosure (e.g., types, formats, and approaches) and compare web-based reports, newsletters to health-care organizations, local newspaper reports, and community meetings - Undertake focus groups with members of the public to investigate views and strategies ( $n=10$ focus groups)

- Conduct trials, with each accreditation domain, of the three most-relevant disclosure models $(\mathrm{n}=$ 30 enrollees)

- Evaluate the utilization of the patient journey technique using ethnographic observations of four accreditation survey teams in each of the three accreditation domains ( $\mathrm{n}=$ 12 survey teams)

- Apply this knowledge to these domains, extending earlier tests of the patient journey method [30] - Triangulate the results and compare and contrast the patient journey method against survey outcomes using standards 
Table 2 Twelve interrelated studies of accreditation-overview of approaches and methods (Continued)

$\begin{array}{ll}\text { 10. Short-notice surveys } & \begin{array}{l}\text { What is the effect of the application } \\ \text { of short-notice surveys? }\end{array} \\ & \begin{array}{l}\text { Examine the use of short-notice } \\ \text { surveys under various circumstances, } \\ \text { including variables such as points in } \\ \text { the accreditation cycle and service } \\ \text { type }\end{array}\end{array}$

- Evaluate ACSAA's experience of

short-notice surveys using key

informant interviews with ACSAA staff

$(n=10)$ and randomly selected agedcare facilities $(n=15)$

- Apply this knowledge to trials with ACHS and AGPAL, extending earlier

tests of short-notice surveys [29]

- Evaluate the ACHS and AGPAL trials and triangulate the data with previous results

\begin{tabular}{ll}
\hline 11. Surveyors and their & What are the roles, effectiveness, and \\
place in accreditation & reliability of surveyors?
\end{tabular}

Conduct an ethnographic analysis of surveyors and surveying processes, with a comparative analysis of the roles, effectiveness and reliability of surveyors in the three accreditation domains

- Analyse existing accreditation databases to assess the relationships between the judgements and survey outcomes of accreditation teams, to quantify the variation between the teams and surveyors

- Undertake experiments, using test scenarios, with the methods developed in earlier research to assess the surveyors' reactions to and consistency in differing accreditation situations [31]

12. Differentiate effective How do effective and less-effective and ineffective uses of organizations use accreditation levers accreditation processes and to improve performance? methods to promote change
Undertake a comparative, randomized, - Examine randomly selected stratified examination of effective and organizations, 20 in each accreditation less-effective organizations and the domain ( $n=60$ organizations) ways they use accreditation to promote performance improvement, drawing upon the results of study 3 .
- Separate these organizations into a split sample of effective and lesseffective organizations, judged by stakeholders' attributions and external organizational performance criteria

- Apply detailed case study methods to derive both quantitative and qualitative indicators; assess how the two samples use accreditation to improve performance and promote change
As shown in Table 2 a wide range of research techniques have been designed and will be applied, including objective empirical measurements, ethnographic observations, focus groups, interviews, trials, ranking exercises, and questionnaire surveys, providing a rich database. This will help create the triangulation effect often missing in discrete, project-based research, which has often produced unrelated, fragmented, and incommensurate findings in the past. A systems approach both to the triangulated multi-method design and to interpreting the findings will be taken, facilitating an understanding of the complex knowledge base that twelve interrelated studies will bring.

\section{Discussion}

We have established a partnership with the main healthcare, general-practice, and aged-care accreditation providers in the country, thereby incorporating the major accreditation domains in the one overarching study. This has allowed us to design policy- and industry-relevant research, e.g., to evaluate current accreditation processes (aim 1: studies 1, 2, 3 and 12) and to improve future accreditation approaches (aim 3: studies, 7-11) (Tables 1 and 2).

There has been no persuasive cost-benefit analysis of accreditation internationally, and we intend to address this oversight in aim 2 via study 6 . Insufficient work has been directed towards the assessment of new methods of accreditation, such as short-notice surveys (i.e., testing their validity) and tracking patients on their journeys through the system, whereby services are assessed based on the quality of care delivered longitudinally. These initiatives require novel assessment methods, e.g., studies 9 and 10 (Table 2).

To address aim 4, we will develop and test a new standard for consumer involvement in accreditation, which will be required for the next generation of accreditation designs [23]. The research technologies, which we will use in unique configurations across the studies, include the Delphi method in study 1, following our use of an earlier version of this in previous research; [40] ethnographic mapping in study 3 , based on our experience in recent research; [41] and randomized designs applied to health-service organizational research in 
studies 2, 5, and 12. A mix of studies of this kind is challenging to do, but is needed given the pervasiveness of accreditation and its lack of an evidence base. ACCREDIT results from the 12 studies will facilitate a systems view of accreditation; given its complexity, this seems highly desirable.

\section{Conclusion}

The ACCREDIT project has been planned in response to questions that the partners, customers of accreditation services, policy bodies (e.g., ACSQHC and CEC), and public and private funders of health-care have raised for many years about the utility, reliability, and cost-effectiveness of accreditation. Our findings are designed to build on what we already know, fill a number of research gaps, and facilitate the improvement of accreditation and the transparency and credibility of the accreditation, surveying and standards-setting processes.

\section{Abbreviations \\ ACCREDIT: Accreditation Collaborative for the Conduct of Research, Evaluation and Designated Investigations through Teamwork; ACHS: The Australian Council on Healthcare Standards; ACSAA: Aged Care and Standards Accreditation Agency; ACSQHC: Australian Commission on Safety and Quality in Health Care; AGPAL: Australian General Practice Accreditation Limited; AlHI: Australian Institute of Health Innovation; CEC: Clinical Excellence Commission; ISO: International Organization for Standardization; MARQulS: Methods of Assessing Response to Quality Improvement Strategies}

\section{Acknowledgements and funding}

This research is supported under Australian Research Council's Linkage Projects scheme (project LP100200586). Human Research Ethics Committee approval for its conduct was granted by the University of New South Wales (HREC 10274). We acknowledge the staff of the industry partners (ACHS, AGPAL, ACSAA) and the quality improvement agencies (ACSQHC, CEC) who provided support for the project. We appreciate Ms Danielle Marks' valuable research assistance in the development of this paper.

\section{Author details}

'University of New South Wales, Kensington, NSW 2052, Australia. ${ }^{2}$ Australian Council on Healthcare Standards, 5 Macarthur Street, Ultimo NSW 2007, Australia. ${ }^{3}$ Quality in Practice/Australian General Practice Accreditation Limited, PO Box 2058, Milton QLD 4064, Australia. ${ }^{4}$ Aged Care Accreditation Agency Limited, PO Box 773, Parramatta NSW 2124, Australia. ${ }^{5}$ Australian Commission on Safety and Quality in Health Care, GPO Box 5480, Sydney NSW 2001, Australia. ${ }^{6}$ Clinical Excellence Commission, GPO Box 1614, Sydney, NSW 2001, Australia. 'University of Technology, 15 Broadway, Ultimo NSW 2007, Australia. ${ }^{8}$ Karolinska Institute, Fakturor, Box 23 109, SE-104 35 Stockholm, Sweden. ${ }^{9}$ University of Southampton, University Road, Southampton SO17 1BJ UK. ${ }^{10}$ Avedis Donabedian University Institute, Autonomous University of Barcelona, CIBER Epidemiology and Public Health (CIBERESP), Spain. ${ }^{11}$ European Society for Quality in Healthcare, St Camillus Hospital, Shelbourne Road, Limerick, Ireland.

\section{Authors' contributions}

JB and JW are the Chief Investigators of the ACCREDIT project. JB, JW, BJ, SC, $\mathrm{MBr}, \mathrm{MBa}, \mathrm{CH}, \mathrm{DG}$ and $\mathrm{MP}$ contributed to developing the overarching research strategy. AC, AG and JC are responsible for specified studies. MW provided statistical advice and expertise. DD supported the development of the research proposal and provided advice on securing ethics approval. DG is senior research fellow working across the projects and MP provides expert advice. $\mathrm{RH}$ and $\mathrm{MM}$ are respectively responsible for qualitative and quantitative research across the projects. $Ј,, C P, R S$ and CS are members of the International Advisory Group, and offered strategic counsel on the program of studies. Team members are responsible, respectively, for leading studies 5, 6 and 7 [JB]; study 12 [JW]; study 1 [B]]; study 3 [SC]; study 2 $[\mathrm{MBr}]$; study 7 [MBa]; study $9[\mathrm{CH}]$; studies 4 and $11[\mathrm{DG}$ and MP]; study 8 $[\mathrm{AC}]$; and study $10[\mathrm{AG}$ and JC]. All authors read and approved the final manuscript.

\section{Competing interests}

The authors declare that they have no competing interests.

Received: 22 February 2011 Accepted: 9 October 2011

Published: 9 October 2011

\section{References}

1. Shaw C, Kutryba B, Braithwaite J, Bedlicki M, Warunek A: Sustainable healthcare accreditation: messages from Europe in 2009. Int J Qual Health Care 2010, 22:341-350.

2. Stimson WA: Better public schools with ISO 9000:2000. Qual Prog 2003, 36:38-45.

3. Skilbeck M, Connell H: Quality Assurance and Accreditation in Australian Higher Education: A National Seminar on Future Arrangements Canberra: Department of Education, Training and Youth Affairs; 2000.

4. Fiekers T, Littler DA, Dale BG: Applying the ISO 9000 series to higher education: an assessment model. International Journal of Continuing Engineering Education and Life-Long Learning 1999, 9:30-44.

5. Jones LG, Price AL: Changes in computer science accreditation. Commun ACM 2002, 45:99-103.

6. Yacout S, Bourbonnais P, Boudreau J: Integrating ISO 9000 with HACCP programs in seafood processing industry. Computers \& Industrial Engineering 1998, 35.

7. Chapleau W: Accreditation of ambulance services: building EMS for the future. Emerg Med Serv 2000, 29:73-75.

8. Gough LA, Reynolds TM: Is Clinical Pathology Accreditation worth it? A survey of CPA-accredited laboratories. British Journal of Clinical Governance 2000, 5:195.

9. Casile M, Davis-Blake A: When accreditation standards change: Factors affecting differential responsiveness of public and private organizations. Acad Manage J 2002, 45:180.

10. Casper S, Hancke B: Global quality norms within national production regimes: ISO 9000 standards in French and German car industries. Organization Studies 1999, 20:961-986.

11. Batalden $P$, Leach D, Swing S, Dreyfus H, Dreyfus S: General competencies and accreditation in graduate medical education. Health Aff 2002, 21:103.

12. Hoyle D, OBrien P: ISO 9000-quality systems handbook. New technology, work and employment 1996, 11:148-150.

13. Corbett CJ, Luca AM, Pan JN: Global perspectives on global standards: a fifteen-economy survey of ISO 9000 and ISO 14000. ISO Management Systems 2003, January-February:31-40.

14. Greenfield D, Braithwaite J: Health sector accreditation research: a systematic review. Int I Qual Health Care 2008, 20:172-183.

15. Pomey MP, Contandriopoulos AP, Francois P, Bertrand D: Accreditation: a tool for organizational change in hospitals? Int I Qual Health Care Assur 2004, 17:113-124.

16. Øvretveit J: Does Improving Quality Save Money? A Review of Evidence of which Improvement to Quality Reduce Costs for Health Service Providers London: The Health Foundation; 2009.

17. Groene O, Klazinga N, Wagner C, Arah O, Thompson A, Bruneau C, Suñol R, DUQuE Research Project: Investigating organizational quality improvement systems, patient empowerment, organizational culture, professional involvement and the quality of care in European hospitals: the 'Deepening our Understanding of Quality Improvement in Europe (DUQuE)' project. BMC Health Serv Res 2010, 10:281.

18. Pomey M-P, Lemieux-Charles L, Champagne F, Angus D, Shabah A, Contandriopoulos A-P: Does accreditation stimulate change? A study of the context and the impact of the accreditation process on Canadian healthcare organisations. Implement Sci 2010, 5:31.

19. Scrivens $E$, Klein R, Steiner A: Accreditation: what can we learn from the Anglophone model? Health Policy 1995, 34:193-204.

20. Greenfield D, Braithwaite J: Developing the evidence base for accreditation of healthcare organisations: a call for transparency and innovation. Qual Saf Health Care 2009, 18:162-163. 
21. Greenfield D, Travaglia J, Braithwaite J, Pawsey M: An analysis of the health sector accreditation literature. A report for the Australian Accreditation Research Network: Examining future health care accreditation research Sydney: Centre for Clinical Governance Research, UNSW; 2007 [http://clingov.med.unsw.edu.au].

22. Commonwealth of Australia: Evaluation of the impact of accreditation on the delivery of quality of care and quality of life to residents in Australian Government subsidised residential care homes Canberra: Commonwealth of Australia; 2007.

23. Braithwaite J, Greenfield D, Westbrook J, Pawsey M, Westbrook M, Gibberd R, Naylor J, Nathan S, Robinson M, Runciman W, Jackson M, Travaglia J, Johnston B, Yen D, McDonald H, Low L, Redman S, Johnson B, Corbett A, Hennessy D, Clark J, Lancaster J: Health service accreditation as a predictor of clinical and organisational performance: a blinded, random, stratified study. Qual Saf Health Care 2010, 19:14-21.

24. Vallejo P, Suñol R: MARQulS: quality improvement strategies for European cross-border healthcare. Qual Saf Health Care 2009, 18:i1-i2.

25. Shaw C, Groene O, Mora N, Suñol R: Accreditation and ISO certification: do they explain differences in quality management in European hospitals. Int J Qual Health Care 2010, October 8.

26. Hindle D, Braithwaite J, Travaglia J, ledema R: Patient safety: a comparative analysis of eight Inquiries in six countries Sydney: Centre for Clinical Governance Research, The University of New South Wales; 2006 [http:// www.med.unsw.edu.au/medweb.nsf/resources/Projects7/\$file/Patient+Safety +report+WEB3.pdf].

27. Braithwaite J, Westbrook J, Pawsey M, Greenfield D, Naylor J, ledema R, Runciman B, Redman S, Jorm C, Robinson M, Nathan S, Gibberd R: A prospective, multi-method, multi-disciplinary, multi-level, collaborative, social-organisational design for researching health sector accreditation. BMC Health Serv Res 2006, 6:113.

28. Dean Beaulieu N, Epstein AM: National Committee on Quality Assurance health-plan accreditation: predictors, correlates of performance, and market impact. Med Care 2002, 40:325-337.

29. Johnston B, Hennessy D, McDonald H, Yen D, Clark S, Fahey K, Greenbank S, Petrakis N, Moody J, Braithwaite J, Greenfield D, Brandon M: Piloting innovative accreditation methodology: short notice surveys-final report Sydney: Australian Council on Healthcare Standards for the Australian Commission on Safety and Quality in Health Care; 2009 [http://www.achs. org.au/].

30. Johnston B, Hennessy D, McDonald H, Yen D, Braithwaite J, Greenfield D: Piloting innovative accreditation methodology: patient journeys-final report Sydney: Australian Council on Healthcare Standards for the Australian Commission on Safety and Quality in Health Care; 2009 [http://www.achs. org.au/].

31. Greenfield D, Pawsey M, Naylor J, Braithwaite J: Are healthcare accreditation surveys reliable? Int J Health Care Qual Assur 2009, 22:105-116.

32. Greenfield D, Braithwaite J, Pawsey M, Johnston B, Robinson M: Distributed leadership to mobilise capacity for accreditation research. J Health Organ Manag 2009, 23:255-267.

33. Lancaster J, Braithwaite J, Greenfield D: Benefits of participating in accreditation surveying. Int J Health Care Qual Assur 2010, 23:141-152.

34. Greenfield D, Braithwaite J: A review of health sector accreditation research literature Sydney: Centre for Clinical Governance Research, UNSW; 2007 [http://clingov.med.unsw.edu.au].

35. Greenfield D, Travaglia J, Braithwaite J, Pawsey M: Unannounced surveys and tracer methodology: literature review. A Report for the Australian Accreditation Research Network: Examining future health care accreditation research Sydney: Centre for Clinical Governance Research, UNSW; 2007 [http://clingov.med.unsw.edu.au].

36. Adrian A, Pawsey M, Braithwaite J, Greenfield D, Travaglia J: Australian Accreditation Research Network Project, final report for the Department of Health and Ageing Sydney: Australian Council on Healthcare Standards and Centre for Clinical Governance Research, University of New South Wales; 2007 [http://clingov.med.unsw.edu.au].

37. Campbell DT, Fiske DW: Convergent and discriminant validation by the multitrait, multimethod matrix. Psychol Bull 1959, 56:81-105.

38. Klein KJ, Kozlowski SWJ: Multilevel Theory, Research and Methods on Organizations: Foundations, Extensions and New Directions San Franciso: Jossey-Bass; 2000.
39. Fink A: Evaluation Fundamentals: Guiding Health Programs, Research and Health Policy Newbury Park: Sage; 1993.

40. Clay-Williams R, Braithwaite J: Determination of health care teamwork training competencies: a Delphi study. Int J Qual Health Care 2009, 21:433-440.

41. Nugus $P$, Braithwaite J: The dynamic interaction of quality and efficiency in the Emergency Department: Squaring the circle? Soc Sci Med 2010, 70:511-517.

42. Brent RJ: Applied Cost-Benefit Analysis. Cheltenham, Edward Elgar 2006.

doi:10.1186/1756-0500-4-390

Cite this article as: Braithwaite et al: Strengthening organizational performance through accreditation research-a framework for twelve interrelated studies: the ACCREDIT project study protocol. BMC Research Notes 2011 4:390.

\section{Submit your next manuscript to BioMed Central and take full advantage of:}

- Convenient online submission

- Thorough peer review

- No space constraints or color figure charges

- Immediate publication on acceptance

- Inclusion in PubMed, CAS, Scopus and Google Scholar

- Research which is freely available for redistribution

Submit your manuscript at www.biomedcentral.com/submit
C Biomed Central 